\title{
Non-tuberculous Mycobacterium species causing mycobacteriosis in farmed aquatic animals of South Africa
}

\author{
Nomakorinte Gcebe ${ }^{1 *} \mathbb{D}$, Anita L. Michel ${ }^{2}$ and Tiny Motlatso Hlokwe ${ }^{1}$
}

\begin{abstract}
Background: Mycobacteriosis caused by non-tuberculous mycobacteria (NTM), is among the most chronic diseases of aquatic animals. In addition, fish mycobacteriosis has substantial economic consequences especially in the aquaculture and fisheries industry as infections may significantly decrease production and trade. Some fish NTM pathogens are highly virulent and zoonotic; as such, infection of aquaria with these pathogens is a public health concern.

In this study, we report isolation of nine different NTM species from sixteen aquatic animals including different fish species, frogs and a crocodile. Given the clinical significance of Mycobacterium marinum and its close relation to Mycobacterium tuberculosis, as well as the significance of ESAT 6 and CFP-10 secretion in mycobacterial virulence, we analysed the esxA and esxB nucleotide sequences of $M$. marinum isolates identified in this study as well as other mycobacteria in the public databases.

Results: Mycobacterium shimoidei, Mycobacterium marinum, Mycobacterium chelonae, Mycobacterium septicum /M. peregrinum and Mycobacterium porcinum were isolated from gold fish, Guppy, exotic fish species in South Africa, koi and undefined fish, Knysna seahorse, as well Natal ghost frogs respectively, presenting tuberculosis like granuloma. Other NTM species were isolated from the studied aquatic animals without any visible lesions, and these include Mycobacterium sp. N845 T, Mycobacterium fortuitum, a member of the Mycobacterium avium complex, and Mycobacterium szulgai. Phylogenetic analysis of mycobacteria, based on esxA and esxB genes, separated slow growing from rapidly growing mycobacteria as well as pathogenic from non-pathogenic mycobacteria in some cases.

Conclusions: Isolation of the different NTM species from samples presenting granuloma suggests the significance of these NTM species in causing mycobacteriosis in these aquatic animals. The study also revealed the potential of esXA and esxB sequences as markers for phylogenetic classification of mycobacteria. Observations regarding use of es $A$ and esxB sequences for prediction of potential pathogenicity of mycobacteria warrants further investigation of these two genes in a study employing NTM species with well-defined pathogenicity.
\end{abstract}

Keywords: Mycobacteriosis, Nontuberculous mycobacteria, Aquatic animals

\footnotetext{
* Correspondence: gceben@arc.agric.za

${ }^{1}$ Tuberculosis Laboratory, Agricultural Research Council - Onderstepoort

Veterinary Research, Onderstepoort, South Africa

Full list of author information is available at the end of the article
}

(c) The Author(s). 2018 Open Access This article is distributed under the terms of the Creative Commons Attribution 4.0 International License (http://creativecommons.org/licenses/by/4.0/), which permits unrestricted use, distribution, and reproduction in any medium, provided you give appropriate credit to the original author(s) and the source, provide a link to the Creative Commons license, and indicate if changes were made. The Creative Commons Public Domain Dedication waiver (http://creativecommons.org/publicdomain/zero/1.0/) applies to the data made available in this article, unless otherwise stated. 


\section{Background}

Non-tuberculous mycobacterial (NTM) infections are among the most common chronic diseases of aquatic animals. NTM are known to infect a number of aquatic animals including fish, amphibians and reptiles [1-5]. NTM infections of fish have been reported in more than 150 species worldwide [6]. The most common pathogens of fish include Mycobacterium marinum, Mycobacterium fortuitum, Mycobacterium chelonae, Mycobacterium peregrinum, Mycobacterium shottsii, Mycobacterium pseudoshottsii and Mycobacterium ulcerans [1, 7-9]. These NTM are of interest as some, particularly $M$. marinum and M. ulcerans, are known to be highly virulent and often result in serious outbreaks in freshwater and aquaria animals. Furthermore, infection of fish with these NTM is a public health concern due to their zoonotic nature. M. marinum causes the so-called 'fish finger's fancier' or fish tank granuloma, or swimming pool granuloma in humans, depending on where the infection was contracted, while $M$. ulcerans causes Buruli ulcer [10]. M. marinum infection in some cases can result in tenosynovitis, arthritis and osteomyelitis [11]. Mycobacterium shottsii and Mycobacterium pseudoshottsii are both phylogenetically related to $M$. marinum and $M$. ulcerans, and were first isolated from a striped bass (Morone saxatilis) [8]. Other NTM like M. fortuitum, M. chelonae and $M$ peregrinum are opportunistic pathogens of fish and humans, especially immuno-compromised individuals [7].

Fisheries and aquaculture remain important sources of food and livelihood for millions of people worldwide. International trade also plays huge socio-economic roles in the aquaculture and fisheries industries, as food security contributor, employment creator, income generator as well as economic growth and development. The aquaculture fish trade industry is reported to have expanded considerably in recent decades [12]. Likewise, reports of mycobacterial infection are on the rise [4, 8, 13-17]. Contrary to other African countries like Nigeria and Uganda, the aquaculture industry in South Africa is still at its infancy. As such, the state has identified this industry as one of the critical areas for promotion. Fish diseases may have substantial economic consequences in the aquaculture industry as infections may significantly decrease production and trade. Control of mycobacterioses in aquaria is by destruction of affected stock and disinfection of the environment as there is no widely used treatment for mycobacterioses in fish [7]. Antibiotic treatment may be a possibility, but is rarely used in the aquaria. The choice of antibiotic is dependent on the infecting NTM species and the strain type [7]. Therefore, monitoring as well as NTM speciation and strain characterization is important in the design of treatment regimens for aquaria mycobacterioses. Currently, in South Africa, limited data is available concerning NTM species circulating in the country's emerging aquaculture industry, let alone the genotypes of each NTM pathogen that may threaten this industry. Isolation of $M$. fortuitum from three fish species originating from fresh water ponds in South Africa was reported in 1990 [7]. In addition, two M. marinum isolates originating from two Anableps anableps (four eyed fish) were used together with isolates from other countries in a study to investigate the genetic diversity and population structure of M. marinum [11].

Virulence profiling is also an important characteristic that may aid in designing improved control measures including development of effective vaccines and drugs. The most studied virulence associated attribute in pathogenic mycobacteria is the ability of the pathogens to subvert host immune defenses characterized by secretion of the components of a specialized system called the type VII secretion system [18]. The mycobacterial type VII secretion system consists of five ESX system (ESX-1 to ESX-5) which were all most likely duplicated from ESX-4 system [19]. ESX-1, ESX-3 and ESX-5 systems have been shown to be involved in protein secretion and are important for virulence of Mycobacterium tuberculosis [20]. The ESX-1 secretion system encoding the region of difference 1 (RD1) (Rv3871Rv3879c) is the most studied. ESAT 6 (early secretory antigenic target -6kDA) and CFP-10 (culture filtrate protein) encoded by esxA (Rv3875) and esxB (Rv 3874), respectively, have been the focus of research for development of vaccines and immunological tests for the diagnosis of tuberculosis in both humans and animals. Several studies have shown that secretion of ESAT 6 and CFP-10 is required for the RD-1 mediated virulence in M. tuberculosis [21, 22].

In this study, we report isolation of NTM from different farmed aquatic animals originating from several aquaria in South Africa. We also determined esx $A$ and $e s x B$ gene sequences of $M$. marinum isolates and further investigated the potential use of these genes in phylogenetic classification of mycobacteria as well as in prediction of potential pathogenicity of NTM.

\section{Results \\ Mycobacterium species isolated from aquatic animals}

Nine NTM species were identified from 16 samples originating from different farmed aquatic species from marine and fresh water environments (Table 1). Mycobacterium shimoidei was isolated from a gold fish (Carassius auratus) intestine and ovary presenting granulomous lesions. Mycobacterium chelonae was isolated from three Knysna seahorses (Hippocampus capensis) originating from the same estuary. One of these seahorses showed a granuloma in the liver and ulceration at the back and under ear, while others did not present any 


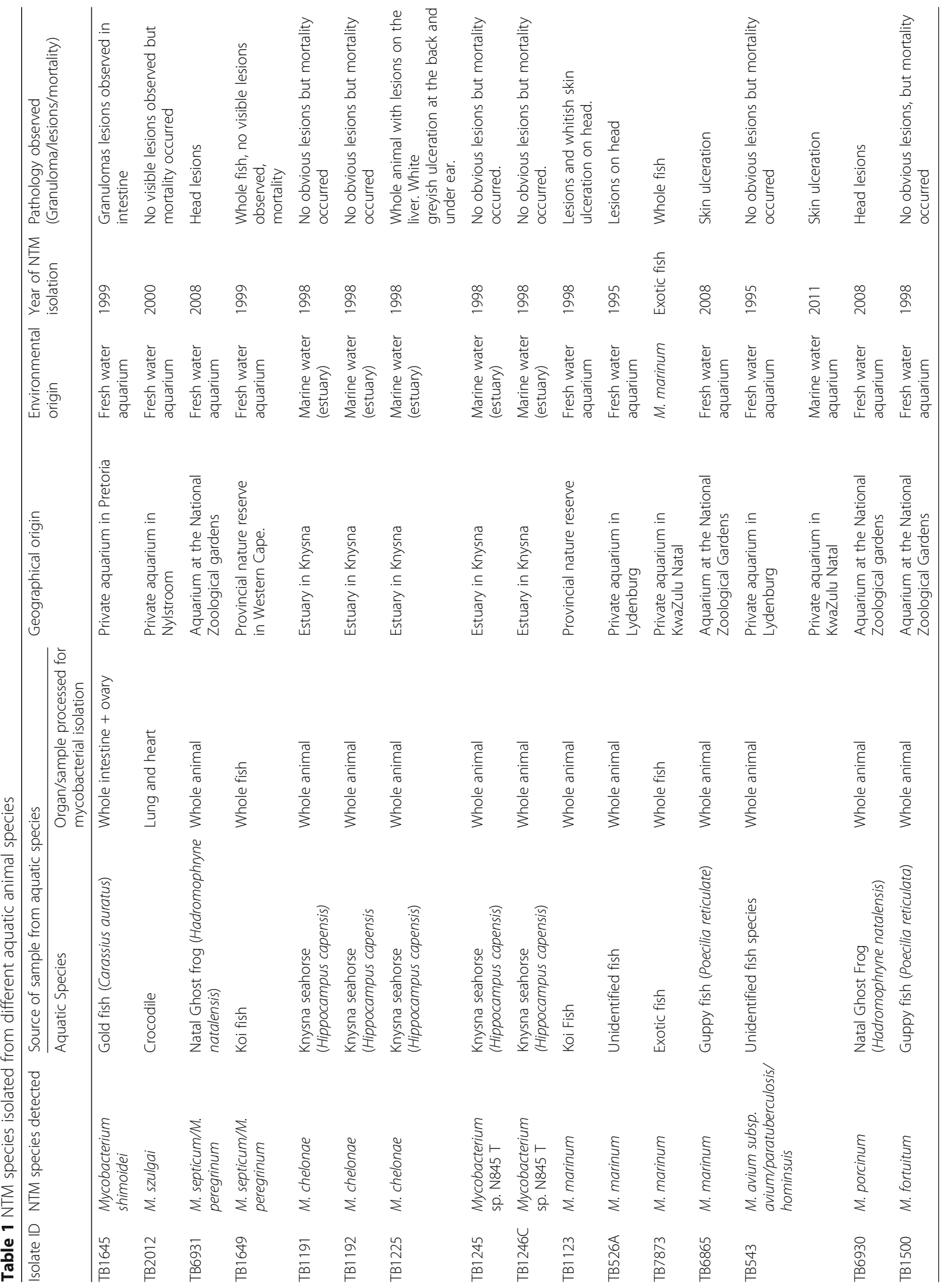


visible lesions. Mycobacterium sp. N845 T was also isolated from two seahorses from the same estuary, presenting no lesions. Mycobacterium fortuitum was isolated from a Guppy fish (Poecilia reticulata) not showing any visible lesions. Mycobacterium septicum/ M. peregrinum was isolated from a koi fish with no visible lesions and a Natal ghost frog with several large granulomas occurring in visceral fat tissue. Mycobacterium porcinum was also isolated from a frog with granuloma in the visceral fat tissue and this frog originated and shared the same aquarium environment as the frog where M. septicum/ M. peregrinum was isolated. Mycobacterium avium complex species was isolated from an unidentified fish species not presenting any obvious lesions. Mycobacterium marinum was isolated from a koi fish, a
Guppy fish as well as an exotic fish in South Africa and an unidentified fish species, all showing skin lesions and or ulcerations on the head. Mycobacterium szulgai was isolated from a crocodile without any obvious lesions (Table 1). The phylogenetic relatedness, of the NTM species identified in this study and those available on the Genbank database is demonstrated in the neighbor-joining tree based on the $16 \mathrm{~S}$ rRNA gene in Fig. 1. The Mycobacterium species identified in this study clustered together with the respective Mycobacterium species available in Genbank.

\section{Mycobacterium esxA and esx $B$ derived phylogeny}

Different mycobacterial housekeeping genes, including $16 \mathrm{~S}$ rRNA, $h s p 65$, sodA and the others have been used

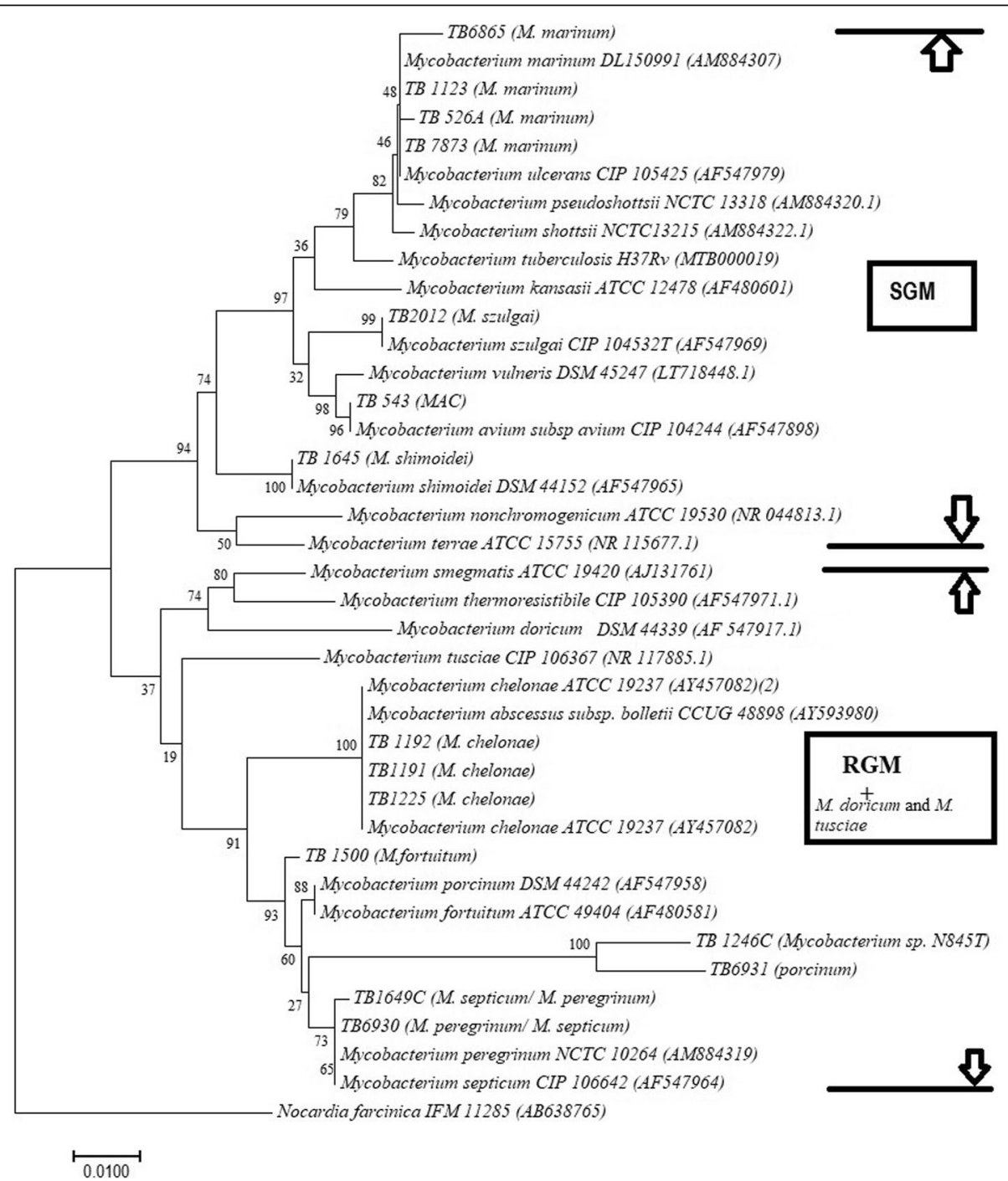

Fig. 1 Phylogenetic tree constructed using neighbour joining method, illustrating the genetic position of the isolates from different aquatic species, based on the partial 165 rRNA gene sequences. Genbank accession numbers for the sequences retrieved from the database are shown in parenthesis. The NTM species identified in this study are also shown in parenthesis next to the isolate identity. The percentage of replicate trees (> 50\%) in which the associated taxa clustered together in the bootstrap test (1000 replicates) are shown next to the branches (Felsenstein, 1985). Nocardia farcinica was used as an out- group sequence. Bar 0.01 substitution per nucleotide 
for species identification and phylogenetic studies. In this study, we inferred the phylogenetic relationship of the $M$. marinum strains identified and those of other Mycobacterium species whose $e s x A$ and $e s x B$ sequences could be retrieved from Genbank (https://www.ncbi.nlm. nih.gov/nucleotide) in order to investigate the use of these two genes in classifying mycobacteria. Mycobacterium marinum strains identified in this study clustered together with Mycobacterium marinum, Mycobacterium liflandii and Mycobacterium ulcerans from Genbank, as illustrated in the neighbor joining trees in Figs. 2 and 3. Neighbor joining trees generated as illustrated in Figs. 2 and 3 clearly separated the slow-growing mycobacteria (SGM) from the rapidly growing mycobacteria (RGM), with the exception of Mycobacterium nonchromogenicum strain NCK 8460, Mycobacterium vulneris, Mycobacterium tusciae and Mycobacterium doricum that are SGM species, which in this analysis clustered with RGM.

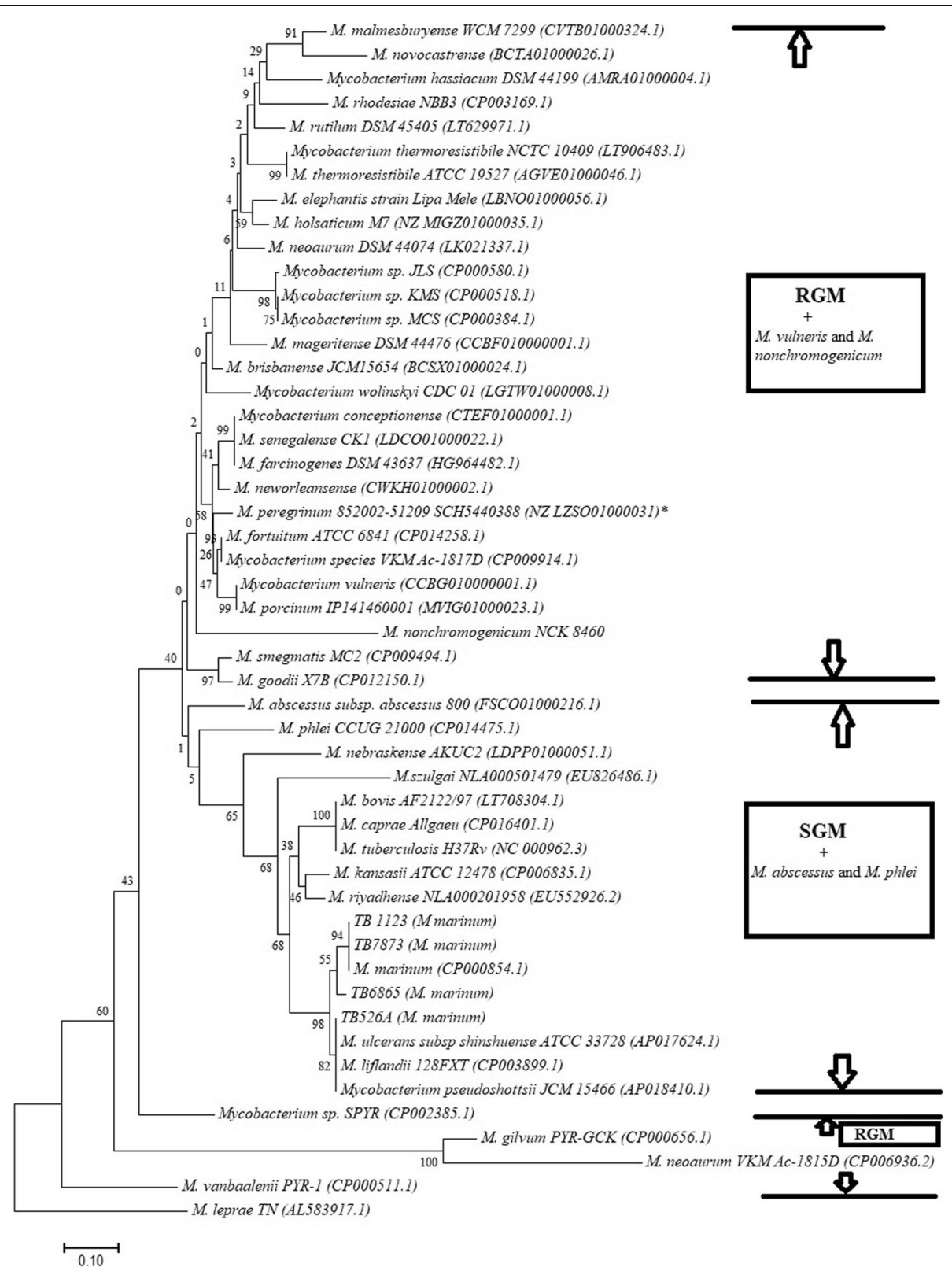

Fig. 2 Phylogenetic tree illustrating the evolutionary history was inferred using the Neighbor-Joining method, based on esxA gene sequences of $M$. marinum strains identified and other Mycobacteria. The evolutionary distances were computed using the Maximum Composite Likelihood method. Genbank accession numbers for the sequences retrieved from the database are shown in parenthesis. The NTM species identified in this study are also shown in parenthesis next to the isolate identity. The percentage of replicate trees $(>50 \%)$ in which the associated taxa clustered together in the bootstrap test (1000 replicates) are shown next to the branches. Evolutionary analyses were conducted in MEGA7 . Bar, 0.1 substitution per nucleotide 


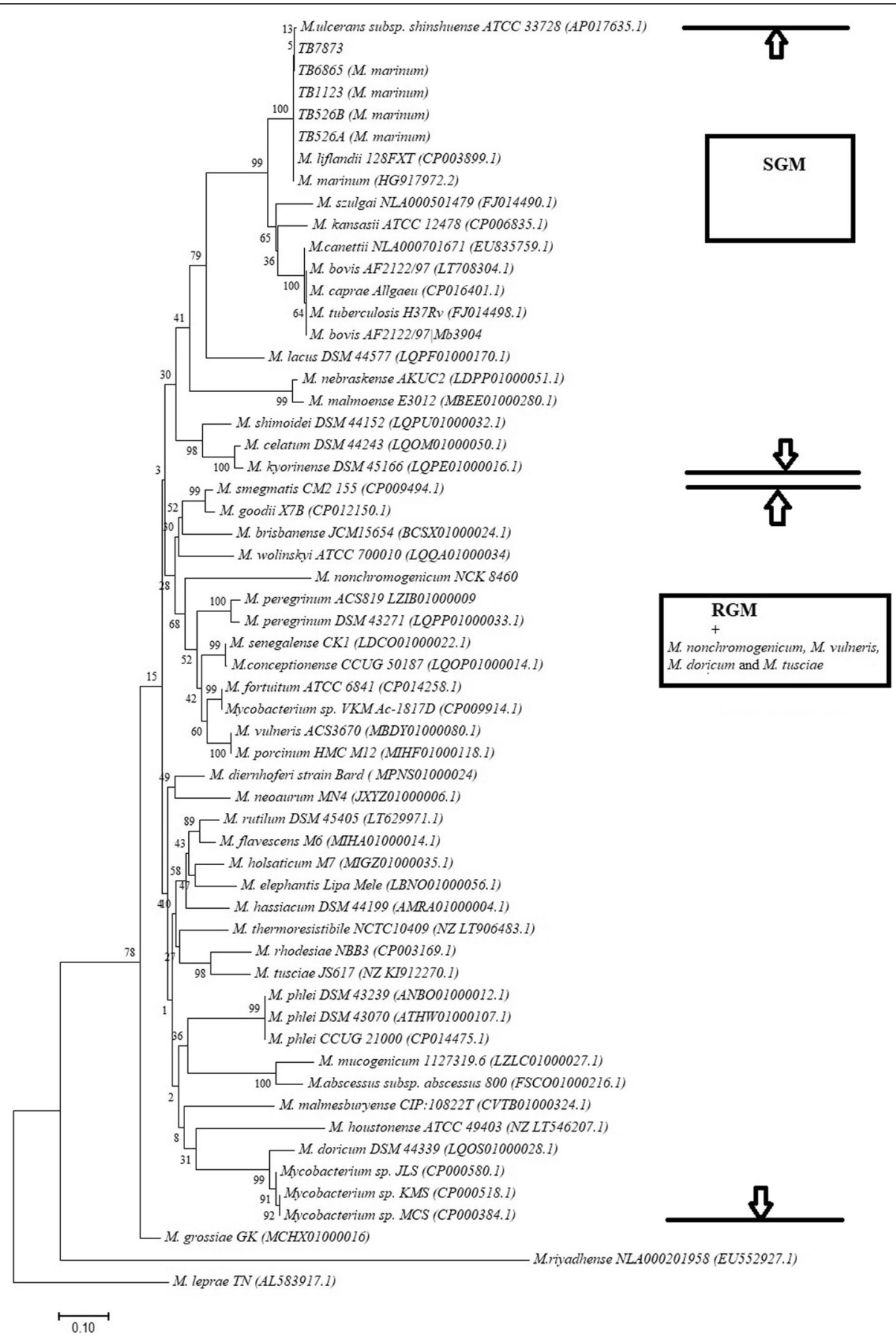

Fig. 3 Phylogenetic tree illustrating the evolutionary history was inferred using the Neighbor-Joining method, based on es $\times B$ gene sequences of $M$. marinum strains identified ana other Mycobacteria. The evolutionary distances were computed using the Maximum Composite Likelihood method. Genbank accession numbers for the sequences retrieved from the database are shown in parenthesis. The NTM species identified in this study are also shown in parenthesis next to the isolate identity. The percentage of replicate trees $(>50 \%)$ in which the associated taxa clustered together in the bootstrap test (1000 replicates) are shown next to the branches. Evolutionary analyses were conducted in MEGA7. Bar, 0.1 substitution per nucleotide

Slowly growing NTM species like M. marinum, M. liflandii, M. ulcerans, M. kansasii, $M$. riyadhense, M. szulgai and $M$. shimoidei clustered together with members of the Mycobacterium tuberculosis complex (MTBC). The RGM species in these trees formed a separate cluster, containing species that are either not known to cause any diseases or maybe opportunistic pathogens at most. The exceptions to this observation are Mycobacterium abscessus subsp. abscessus and Mycobacterium phlei that are RGM but in the phylogenetic tree based on esxA both clustered with SGM. 


\section{Discussion}

Mycobacteriosis is among the most chronic diseases affecting aquatic animals $[3-5,13]$. In this study, sixteen Mycobacterium isolates originating from different species of fish, frogs and a crocodile were characterized by $16 \mathrm{~S}$ rRNA and hsp65 gene sequence analysis for speciation. All these isolates belonged to nine NTM species namely Mycobacterium shimoidei, Mycobacterium marinum, Mycobacterium chelonae, Mycobacterium fortuitum, Mycobacterium szulgai, Mycobacterium septicum/ M. peregrinum, Mycobacterium porcinum and Mycobacterium sp. N845 T. M. shimoidei was isolated strictly from a gold fish intestine (Carassius auratus), originating from a fresh water aquarium, presenting granulomous lesions. M. shimoidei was first isolated in 1972 from a patient with a lung infection and since then several other human cases of this NTM have been reported, suggesting that it might be an emerging pathogen of humans [23-25]. To the best of our knowledge, isolation of this NTM from a gold fish presenting mycobacteriosis granuloma is first to be reported in this study. This therefore could potentially be an addition to opportunistic NTM pathogens of fish.

Mycobacterium marinum and Mycobacterium chelonae are known pathogens of fish [26]. In this study, these NTM species were isolated from different fish species, with $M$. chelonae strictly occurring in sea horses, and M. marinum was detected from a Guppy, koi, exotic fish in South Africa and an undefined fish species showing granuloma. The occurrence of M. marinum in four different fish species from both fresh and marine water environments, suggested the adaptation of Mycobacterium marinum in different water environments and fish species. In South Africa, a case of $M$. marinum infection was last reported in 1987 as Fish tank granuloma [27]. Given its zoonotic nature and its phylogenetic relatedness to Mycobacterium tuberculosis complex, it was therefore pertinent that we characterise the $M$. marinum isolates beyond species identification. Virulence profiling is important in the characterisation of microorganisms. Virulence factors in pathogenic mycobacteria are those attributes that enable the bacterium to infect, survive and multiply in host macrophages, resulting in disease symptoms [28]. Albeit the host mounting complex immune responses that often sequesters the pathogen in granuloma, the key to Mycobacterium virulence lies in, at least in part with, their ability to establish residence and proliferate inside the macrophages [29]. There is now strong evidence that the ESX-1 secretion system of mycobacteria encoding RD1 is essential for virulence in members of $M$. tuberculosis complex. RD1 mediated virulence is the most studied virulence associated attribute in mycobacteria. Several studies have demonstrated the role of esxA and esxB in mycobacterial pathogenesis.
Some studies have demonstrated an attenuated phenotype in mouse models of infection following inactivation of $e s x A$ and $e s x B$ or other genes of the ESX-1 secretion system of $M$. tuberculosis [30-32]. These and many other studies thus confirm the significance of ESX-1 secretion system in Mycobacterium tuberculosis virulence. Furthermore, some studies have reported loss of ESX-1 secretion, impaired bacterial growth of $M$. marinum in macrophages and reduced bacterial virulence in zebra fish models of infection, resulting from inactivation of either es $x A$ or $e s x B$ as well as transposon mutagenesis in various genes encoded or adjacent the RD1 locus. [33, 34]. Some non-pathogenic NTM, however, harbor $e s x A$ and $e s x B$ genes in their genomes with different sequences, whose functions are unknown $[18,35]$. For this reason, we set out to investigate, whether sequence differences in es $x A$ and $e s x B$ genes of different mycobacteria could be used in their phylogenetic classification, as well as prediction of their potential pathogenicity. Sequence analysis of esxA and $e s x B$ of the four fish $M$. marinum strains isolated in this study were determined using a PCR-sequencing assay. Phylogenetic analysis of these strains, as well as other NTM that harbor esxA and esxB, and members of the Mycobacterium tuberculosis complex, revealed phylogenetic profiling that separated SGM, most of which are pathogenic mycobacteria, and RGM which are either not known to be pathogenic or are opportunistic pathogens at most. Mycobacterium nonchromogenicum strain NCK 8460, a soil isolate that is SGM [35], and other SGM namely, Mycobacterium vulneris, Mycobacterium tusciae and Mycobacterium doricum were exceptions to this observation since they were found to be phylogenetically close to the RGM clusters in the esxA and esxB trees as indicated in Figs. 2 and $3 M$. doricum and $M$. tusciae were also found to be close to RGM in the phylogenetic analysis based on the 16S rRNA gene as indicated in Fig. 1. Other exceptions were $M$. abscessus subsp. abscessus and $M$. phlei, which though they are RGM clustered with SGM species in the es $x A$ tree. Therefore, es $x A$ and es $x B$ may be used as markers for phylogenetic classification of mycobacteria by growth rate to some extent with some exceptions for example $M$. nonchromogenicum, M. vulneris, $M$. tusciae, M. doricum, M. phlei and M. abscessus subsp. abscessus as seen in this study. We have noted that prediction of pathogenicity of NTM based on esxA and es $x B$ sequences, as markers, may not result to a definite ruling, as highlighted in this study. We observed that NTM that were previously reported as opportunistic pathogens, were found in some cases to be closer to NTM that are not known to be pathogenic. On the other hand opportunistic RGM pathogens like $M$. abscessus subsp. abscessus and $M$. phlei were found to be phylogenetically closer to known pathogenic SGM, suggesting that perhaps in this case es $x A$ 
and es $x B$ sequences may be markers for prediction of pathogenicity in NTM. Likewise, some of the nonpathogenic SGM such as Mycobacterium nonchromogenicum were found to cluster with non-pathogenic RGM. The established pathogenic NTM, like M. marinum and $M$. kansasii, clustered with members of the MTBC, whose pathogenicity is not questioned, in the phylogenetic analysis based on the esxA and esxB (Figs. 2 and 3). Indeed, observation by van Ingen et al, based on identical sequences of two strains of $M$. szulgai that were clinically relevant or non-relevant, suggested that the gene sequences do not provide a complete picture of pathogenicity in NTM [36]. This suggests that studies that are more extensive on this topic, employing both pathogenic and non-pathogenic Mycobacterium species with welldefined pathogenicity are needed to further investigate the association of esxA and esxB sequence homologies to pathogenicity of mycobacteria. As van Ingen and coworkers previously recommended, description of new species should consider sequence analysis of genes of ESX-1, in particular esxA and esxB. It should, however, be noted that ESX-1 region is not the only virulence factor in mycobacteria. For instance, some pathogenic members of the Mycobacterium avium complex and Mycobacterium microti lack this region [19, 37]. However, the potential use of these two genes as markers to predict the potential pathogenic nature of NTM should not be ruled out.

Although M. chelonae is a known pathogen of fish, we report its occurrence and its potential role in disease causation in South Africa's estuary Knysna seahorses for the first time in this study. We also identified Mycobacterium sp. N845 T in Knysna seahorses from the same estuary where an outbreak of mycobacterial disease occurred. This is not a validly published Mycobacterium species, but it is phylogenetically closely related to Mycobacterium fortuitum and Mycobacterium porcinum as determined by its partial 16S rRNA gene sequence (Fig. 1). We also identified Mycobacterium septicum/M. peregrinum as well as Mycobacterium porcinum each in Natal ghost frogs showing disease symptoms. The two ghost frogs originated from the same fresh water aquarium. A recent study conducted in South Africa reported isolation of $M$. abscessus- chelonae complex from frogs in captivity [5]. Except for a few cases reported in laboratory animals, like those of Mycobacterium liflandii and Mycobacterium marinum, mycobacteriosis in frogs is rare [38]. Mycobacterium septicum, M. peregrinum and Mycobacterium porcinum are all known opportunistic pathogens of humans and livestock [26]. Their isolation in amphibians in this study demonstrate the ubiquity of these NTM species as well as their ability to cause disease in a Natal ghost frog. Mycobacteriosis is very rarely reported in crocodiles. In this study, Mycobacterium szulgai was isolated from a crocodile sample with tuberculosis like lesions, demonstrating its role in causing disease in aquatic animal. M. szulgai is now becoming a common NTM pathogen of humans and has been reported to cause diseases in animals as well [39]. M. szulgai infection manifest as both pulmonary and extra pulmonary, although pulmonary disease is the most common manifestation and it may clinically and radiologically resemble pulmonary tuberculosis [40]. As such, isolation of $M$. szulgai is considered clinically significant. Mycobacterium avium and Mycobacterium ulcerans have been reported to cause generalised granuloma in Crocodylus johsonii and Crocodylus niloticus respectively [41]. Isolation of a Mycobacterium avium complex species from fish demonstrates the ubiquity of these NTM species, which are known opportunistic pathogens of humans and have been isolated from several animal species and the environment.

\section{Conclusion}

In conclusion, we have detected NTM causing mycobacterioses in fish, frogs and a crocodile. Some of these NTM, like $M$. marinum and $M$. chelonae are known pathogens of aquatic animals. Reports of $M$. shimoidei infections in fish are scarce and this NTM is reported for the first time in a gold fish (Carassius auratus) in South Africa. This study contributes knowledge about the NTM species causing mycobacterioses in farmed aquatic animals in our country. Even though the study provide limited information regarding NTM diversity in aquatic animals, it does provide insights into the occurrence of pathogenic NTM that may threaten the emerging aquaculture industry in South Africa. Indeed, previous studies conducted country wide to investigate the prevalence of NTM in cattle, buffaloes and other wildlife species, as well as the environment in South Africa, found that NTM are highly abundant and more diverse than in many other parts of the world $[42,43]$. This and the previous studies raise serious economic and public health concerns as some NTM isolated have a zoonotic potential, hence, warrants proper monitoring programs of animal mycobacteriosis for control purposes. Sequence differences of $e s x A$ and $e s x B$ in different mycobacteria were shown in this study to have a potential use in phylogenetic classification of mycobacteria and in prediction of the potential pathogenicity of NTM species.

\section{Methods}

\section{Sample origin}

Samples used in this study were obtained from routine diagnostic submissions to the Tuberculosis Laboratory of the Agricultural Research Council-Onderstepoort Veterinary Research (ARC-OVR) from different regions of South Africa. Routine submissions at the Tuberculosis 
laboratory form part of the State Veterinary Service's strategy for confirming mycobacterial infections in animals. Samples were obtained from the following aquatic animals: crocodile, Natal ghost frog and different species of fish collected during the years 1995-2011. A summary of the animal sample sources including geographic origin, animal species and sample type is presented in Table 1.

\section{Laboratory examination, Mycobacterium isolation and identification}

Laboratory examination of the sample to be cultured was performed macroscopically to determine the presence or absence of tuberculous lesions/ granulomas and findings were recorded. Tissue samples as well as whole animals (in case of seahorses, gold fish and frogs) were processed and cultured according to standard laboratory procedures as described by Gcebe and Hlokwe as well as Hlokwe et al., [43, 44]. Ziehl-Neelsen staining was performed to determine the acid fastness of colonies typical of Mycobacterium species, which were selected, based on morphology. The acid-fast isolates were stored at $-20{ }^{\circ} \mathrm{C}$ for further molecular analysis.

\section{DNA extraction}

DNA was extracted using the heating method [44]. Briefly, individual acid-fast colonies were suspended in $100 \mu \mathrm{l}$ of sterile distilled water and heated at $95{ }^{\circ} \mathrm{C}$ in a heating block/ or in boiling water for $25 \mathrm{~min}$. The culture lysates were stored at $-20{ }^{\circ} \mathrm{C}$ or $-70{ }^{\circ} \mathrm{C}$ until further analysis. The culture lysates were used as DNA template in subsequent PCR protocols.

\section{In vitro amplification and sequencing of the} mycobacterial $16 \mathrm{~S}$ rDNA and $h s p 65$ genes for speciation PCR and sequencing of the 577 bp fragment of the mycobacterial 16S rRNA gene was used for mycobacterial speciation $[42,45]$. Sequencing of the 439 bp hsp 65 gene fragment was done for differentiation of some NTM species with similar, or identical $16 \mathrm{~S}$ rRNA, which could not be delineated using the $16 \mathrm{~S}$ rRNA gene PCR-sequencing assay [43]. Sequence analysis of the $h s p 65$ gene fragments was performed to differentiate Mycobacterium chelonae and Mycobacterium abcessus group as well as Mycobacterium marinum and Mycobacterium ulcerans. Amplification and sequence analysis of both gene fragments were done as described by Gcebe et al., [35].

\section{Amplification of esxA and esxB genes of M. marinum isolates}

Crude DNA extracts were used as template DNA in PCR protocols targeting es $x A$ and es $x B$ genes. The following primer pairs: esat F: 5'ATGACAGAGCAGCAG TGGAA 3' and esat R 5'CTATGCGAACATCCCAGT
GA3' located at positions $1-20$ and 288-271, respectively, of the $M$. marinum esxA (MMAR_5450) gene sequence were used for amplification of the es $x A$ gene. Primers cfp-10F 5'ATGGCAGAGATGAAGACCGA3' and cfp-10 R 5' TCAGAAGCCCATTTGCGAGG 3' located at positions $1-20$ and positions 303-284 of the $M$. marinum cfp-10 (MMAR_5449) were used for the in vitro amplification of es $x B$ gene sequences. The $M$. marinum es $x A$ and esxB gene sequences used to manually design these primers were retrieved from the Marinolist database (http://svitsrv8.epfl.ch/mycobrowser/marinolist.html).

\section{Phylogenetic analysis of Mycobacterium species}

To determine the phylogenetic relationship among all the Mycobacterium species identified in this study as well as between these species and the other published species, a neighbor joining tree based on the partial $16 \mathrm{~S}$ rRNA (577 bp) gene sequences was constructed. Multiple sequence alignments were performed using MEGA version 7.0 platform [46]. Sequences were first trimmed at both the $5^{\prime}$ and $3^{\prime}$ ends, so that all sequences start and end at the same nucleotide position. Phylogenetic relationship of the isolates and other Mycobacterium species was investigated in phylogenetic trees constructed using the neighbor-joining method [47]. The neighbour- joining trees were validated using the maximum composite likelihood method and one thousand bootstrap replicates were run. Nocardia farcinica was used as an outgroup sequence.

Similarly, neighbor joining trees based on es $x A$ and es $x B$ gene sequences were constructed using the sequences of M. marinum isolates from this study as well as those of other mycobacteria. This was done to determine the position of the isolated $M$. marinum strains in the phylogenetic tree as well as to investigate if phylogeny could give insight into the association of the es $x A$ and es $x B$ sequences with pathogenicity of mycobacteria as well as growth rate. The es $x A$ and $e s x B$ gene sequences of different Mycobacterium species were retrieved from Genbank database (https://www.ncbi.nlm.nih.gov/nucleotide), while the es $x A$ and es $x B$ sequences of Mycobacterium nonchromogenicum NCK 8640 was determined in the previous study describing the whole genome of this isolate [35].

The genetic relationship among the $M$. marinum isolates were inferred from the MIRU-VNTR using the BIONUMERICS software version. 7. 6 (www.appliedmaths.com) applying the unweighted group method with arithmetic average (UPGMA).

\section{Genbank nucleotide accession numbers}

Nucleotide sequences of the following gene fragments: 16S rRNA, hsp65, esxA and es $x B$ for the four Mycobacterium marinum strains as well as the $16 \mathrm{~S}$ rRNA and hsp65 for Mycobacterium shimoidei were submitted to 
Genbank (www.Ncbi.nlm.nih.gov/Genbank). The accession numbers for the 16Sr RNA genes are, MF402009, MF402010, MF402011, MF402012 and MF402013, for Mycobacterium shimoidei strain 1645, Mycobacterium marinum strain 1123, Mycobacterium marinum strain 526A, Mycobacterium marinum strain 6865, and Mycobacterium marinum strain 7873 respectively. The accession numbers for $h s p 65$ are MF411150, MF411146, MF411147, MF411148, and MF411149 for Mycobacterium shimoidei strain 1645, Mycobacterium marinum strain 1123, Mycobacterium marinum strain 526A, Mycobacterium marinum strain 6865 and Mycobacterium marinum and strain 7873 respectively. The accession numbers for esxA are, MF411157, MF411153, MF411151 and MF411152 for Mycobacterium marinum strain1123, Mycobacterium marinum strain 526A, Mycobacterium marinum strain 6865 and Mycobacterium marinum strain 7873 respectively. The accession numbers for es $x B$ are, MF411158, MF411154, MF411155 and MF411156 for Mycobacterium marinum strain 1123, Mycobacterium marinum strain 526A, Mycobacterium marinum strain 6865 and Mycobacterium marinum strain 7873 respectively.

\section{Abbreviations \\ CFP-10: 10 kDa Culture filtrate protein; ESAT-6: 6 kDa Early secretory antigenic target; NTM: Non tuberculous mycobacteria; PCR: Polymerase Chain Reaction}

\section{Acknowledgements}

The authors would like to thank Dr. Mouton and Dr. Huchzermeyer for collection of some of the samples from fish, crocodile and frogs as well as for preservation of the NTM isolates. We also thank Ms. B Phahladira for critically reviewing the manuscript and the Agricultural Research Council-Onderstepoort Veterinary Research for financial support.

\section{Funding}

This work was supported by the Agricultural Research Council (Project P10000058) awarded to the Tuberculosis Laboratory Diagnostics at Onderstepoort Veterinary Research. The two authors that carried out the study: TH, and NG are employees of the Agricultural Research Council. The funding body itself had no role in the design of the study and collection, analysis, and interpretation of data and writing the manuscript.

\section{Availability of data and materials}

Nucleotide sequences of the different genes analyzed in this study are available in Genbank (www.Ncbi.nlm.nih.gov/Genbank). The Genbank accession numbers for the different nucleotide sequences are: MF402009, MF402010, MF402011, MF402012, MF402013, MF411150, MF411146, MF411147, MF411148, MF411149, MF411157, MF411153, MF411151, MF411152, MF411158, MF411154, MF411155 and MF411156.

\section{Authors' contributions}

ALM participated in specimen collection and preservation as well as review of the manuscript, TH, participated in data collection, specimen collection, bench work, result analysis and drafting of the manuscript. NG designed the study, collected data, performed bench work, result analysis and drafted the manuscript. All authors have read and approved the manuscript.

\section{Ethics approval and consent to participate}

This study complies with national guidelines of South Africa for routine surveillance/ diagnosis of tuberculosis and other mycobacterioses diseases [Department of Agriculture, Fisheries and Forestry (DAFF) SOP 002; Animal Diseases Act 35 of 1984]. As such no ethics approval was needed for this study as samples were submitted for routine diagnosis.

\section{Competing interests}

The authors declare that they have no competing interests. TH and NG are employees of the Agricultural Research Council.

\section{Publisher's Note}

Springer Nature remains neutral with regard to jurisdictional claims in published maps and institutional affiliations.

\section{Author details}

${ }^{1}$ Tuberculosis Laboratory, Agricultural Research Council - Onderstepoort Veterinary Research, Onderstepoort, South Africa. ${ }^{2}$ Department of Veterinary Tropical Diseases, Bovine Tuberculosis and Brucellosis Research Programme, Faculty of Veterinary Science, University of Pretoria, Onderstepoort, South Africa.

Received: 14 August 2017 Accepted: 5 April 2018

Published online: 13 April 2018

\section{References}

1. Novotny L, Halouzka R, Matlova L, Vavra O, Bartosova L, Slany M, et al. Morphology and distribution of granulomatous inflammation in freshwater ornamental fish infected with mycobacteria. J Fish Dis. 2010;33:947-55.

2. Slany M, Knotek Z, Skoric M, Knotkova Z, Svobodova J, Mrlik V, Moravkova M, Pavlik I. Systemic mixed infection in a brown caiman (Caiman crocodilus fuscus) caused by Mycobacterium szulgai and M. chelonae: a case report. Vet Med. 2010;55:91-6.

3. Willson SJ, Kaufman MG, Merritt RW, Williamson HR, Malakauskas DM, Benbow ME. Fish and amphibians as potential reservoirs of Mycobacterium ulcerans, the causative agent of Buruli ulcer disease. Infect Ecol Epidemiol. 2013;3:19946.

4. Woodhouse SJ, Fitzgerald SD, Lim A, Bolin SR. Disseminated Mycobacterium haemophilum infection in an Assam trinket snake (Elaphe frenata). J Zoo Wildl Med. 2014;45:966-9.

5. Barrows M, Koeppel K, Michel A, Mitchell E. Mycobacterial arthritis and synovitis in painted reed frogs (Hyperolius marmoratus). J Comp Pathol. 2017:156:275-80.

6. Decostere A, Hermans K, Haesebrouck F. Piscine mycobacteriosis: a literature review covering the agent and the disease it causes in fish and humans. Vet Microbiol. 2004;99:159-66.

7. Gauthier TD, Rhodes MW. Mycobacteriosis in fishes: a review. Vet J. 2009;180:33-47.

8. Stine CB, Kane AS, Baya AM. Mycobacteria isolated from Chesapeake Bay fish. J Fish Dis. 2010;33:39-46.

9. Nakanaga K, Hoshino Y, Hattori Y, Yamamoto A, Wada S, Hatai K, Makino M, Ishii N. Mycobacterium pseudoshottsii isolated from 24 farmed fishes in western Japan. J Vet Med Sci. 2012;74:275-8.

10. Sizaire V, Nackers F, Comte E, Portaels F. Mycobacterium ulcerans infection: control, diagnosis, and treatment. Lancet Infect Dis. 2006;31:288-96.

11. Broutin V, Bañuls AL, Aubry A, Keck N, Choisy M, Bernardet JF, et al. Genetic diversity and population structure of Mycobacterium marinum: new insights into host and environmental specificities. J Clin Microbiol. 2012;50:3627-34. https://doi.org/10.1128/JCM.01274-12.

12. FAO. Contributing to food security and nutrition for all. Rome: The State of World Fisheries and Aquaculture; 2016. pp. 200.

13. Dos Santos NM, Do Vale A, Sousa MJ, Silva MT. Mycobacterial infection in farmed turbot Scophthalmus maximus. Dis Aquat Org. 2002;52:87-91.

14. Passantino A, Macri D, Coluccio P, Foti F, Marino F. Importation of mycobacteriosis with ornamental fish: medico-legal implications. Travel Med Infect Dis. 2008:6:240-2444

15. Bozzetta E, Varello K, Giorgi I, Fioravanti ML, Pezzolato M, Zanoni RG, Prearo M. Mycobacterium marinum infection in a hybrid striped bass farm in Italy. J Fish Dis. 2010;33:781-5.

16. Finkelstein $\mathrm{R}$, Oren I. Soft tissue infections caused by marine bacterial pathogens: epidemiology, diagnosis, and management. Curr Infect Dis Rep. 2011;13:470.

17. Imajoh M, Sugiura H, Hashida Y, Hatai K, Oshima SI, Daibata M, Kawai K. Genotypic characteristics of a Mycobacterium sp. isolated from yellowtail Seriola quinqueradiata and striped jack Pseudocaranx dentex in Japan. Microbiol Immunol. 2013;57:13-20.

18. Majlessi L, Prados-Rosales R, Casadevall A, Brosch R. Release of mycobacterial antigens. Immunol Rev. 2015;264:25-45. 
19. van Pittius NC, Sampson SL, Lee H, Kim Y, Van Helden PD, Warren RM. Evolution and expansion of the Mycobacterium tuberculosis PE and PPE multigene families and their association with the duplication of the ESAT-6 (esx) gene cluster regions. BMC Evol Biol. 2006;6:95. https://doi.org/10.1186/ 1471-2148-6-95.

20. Shah S, Briken V. Modular organization of the ESX-5 secretion system in Mycobacterium tuberculosis. Frontiers Cell Infect Microbiol. 2016;6. https://doi.org/10.3389/fcimb.2016.00049.

21. Pym AS, Brodin P, Brosch R, Huerre M, Cole ST. Loss of RD1 contributed to the attenuation of the live tuberculosis vaccines Mycobacterium bovis BCG and Mycobacterium microti. Mol Microbiol. 2002;46:709-17.

22. Bitter W, Houben ENG, Botai D, Brodin P, Brown EJ, Cox JS, et al. Systematic genetic nomenclature for type VII secretion systems. PLoS Pathog. 2009;5: 10. e1000507. https://doi.org/10.1371/journal.ppat.1000507.

23. Tsukamura M. Mycobacterium shimoidei sp. nov., nom. Rev., a lung pathogen. Int J Syst Evol Microbiol. 1982;32:67-9.

24. Koukila-kÄhkÖIÄ P, Paulin L, Brander E, Jantzen E, Eho-remes M, Katila ML. Characterisation of a new isolate of Mycobacterium shimoidei from Finland. J Med Microbiol. 2000;49:937-40.

25. Kanaji N, Kushida Y, Bandoh S, Ishii T, Haba R, Tadokoro A, Watanabe N, Takahama T, Kita N, Dobashi H, Matsunaga T. Membranous glomerulonephritis associated with Mycobacterium shimoidei pulmonary infection. Am J Case Rep. 2013;14:543.

26. Bercovier $\mathrm{H}$, Vincent $\mathrm{V}$. Mycobacterium infections in domestic and wild animals due to Mycobacterium marinum, M. fortuitum, M. chelonae, $M$, porcicum, $M$. farcinogenes, M. smegmatis, M. scrofulaceum, M. xenopi, M. kansasii, M. simiae and M. genavense. Rev Sciet Tech Off Int Epiz. 2001;20:265-90.

27. Mousdicas N, Saxe N. Fish tank granuloma. SA Med J. 1987;71:321-2.

28. Collins DM. Virulence factors of Mycobacterium bovis. Tuberculosis. 2001; 81:97-102.

29. Bigi F, Gioffré A, Klepp L, Santangelo MP, Velicovsky CA, Giambartolomei $\mathrm{GH}$, et al. Mutation in the P36 gene of Mycobacterium bovis provokes attenuation of the bacillus in a mouse model. Tuberculosis. 2005;85:221-6.

30. Hsu T, Hingley-Wilson SM, Chen B, Chen M, Dai AZ, et al. The primary mechanism of attenuation of bacillus Calmette-Guerin is a loss of secreted lytic function required for invasion of lung interstitial tissue. PNAS. 2003;100:12420-5.

31. Stanley SA, Raghavan S, Hwang WW, Cox JS. Acute infection and macrophage subversion by Mycobacterium tuberculosis require a specialized secretion system. PNAS. 2003;100:13001-6.

32. Guinn KM, Hickey MJ, Mathur SK, Zakel KL, Grotzke JE, Lewinsohn DM, et al. Individual RD1-region genes are required for export of ESAT-6/CFP-10 and for virulence of Mycobacterium tuberculosis. Mol Microbiol. 2004;51:359-70.

33. Gao LY, Guo S, McLaughlin B, Morisaki H, Engel JN, Brown EJ. A mycobacterial virulence gene cluster extending RD1 is required for cytolysis, bacterial spreading and ESAT-6 secretion. Mol Microbiol. 2004;53:1677-93.

34. Tan T, Lee WL, Alexander DC, Grinstein S, Liu J. The ESAT-6/CFP-10 secretion system of Mycobacterium marinum modulates phagosome maturation. Cell Microbiol. 2006;8:1417-29.

35. Gcebe N, Michel A, van Pittius NC, Rutten V. Comparative genomics and proteomic analysis of four non-tuberculous Mycobacterium species and Mycobacterium tuberculosis complex: occurrence of shared immunogenic proteins. Frontiers Microbiol. 2017;7:795. https://doi.org/10.3389/fmicb.2016.00795.

36. van Ingen J, de Zwaan R, Dekhuijzen R, Boeree $M$, van Soolingen D. Region of difference 1 in nontuberculous Mycobacterium species adds a phylogenetic and taxonomical character. Bacteriol. 2009;191:5865-7.

37. Brodin P, Eiglmeier K, Marmiesse M, Billault A, Garnier T, Niemann S. Bacterial artificial chromosome-based comparative genomic analysis identifies Mycobacterium microti as a natural ESAT-6 deletion mutant. Infect Immun. 2002;70:5568-78.

38. Suykerbuyk P, Vleminckx K, Pasmans F, Stragier P, Ablordey A, Tran HT, Portaels F. Mycobacterium liflandii infection in European Colony of Silurana tropicalis. Emerg Infect Dis. 2007;13:743-6.

39. Lacasse C, Terio K, Kinsel MJ, Farina LL, Travis DA, Greenwald R. Two cases of atypical mycobacteriosis caused by Mycobacterium szulgai associated with mortality in captive African elephants (Loxodonta africana). J Zoo Wildl Med. 2007;38:101-7.

40. Kim JJ, Lee J, Jeong SY. Mycobacterium szulgai pulmonary infection: case report of an uncommon pathogen in Korea. Korean J Radiol. 2014;15:651-4.

41. Huchzermeyer FW. Diseases of farmed crocodiles and ostriches. Rev Off Int Epizoot. 2002;21:265-76.
42. Gcebe N, Rutten V, Gey van Pittius NC, Michel A. Prevalence and distribution of non-tuberculous mycobacteria (NTM) in cattle, African buffaloes (Syncerus caffer) and their environments in South Africa. Transbound Emerg Dis. 2013;60:74-84.

43. Gcebe N, Hlokwe TM. Non-tuberculous mycobacteria in south African wildlife: neglected pathogens and potential impediments for bovine tuberculosis diagnosis. Frontiers Cell Infect Microbiol. 2017;7:15.

44. Hokwe TM, Van Helden P, Michel AL. Evidence of increasing intra and interspecies transmission of Mycobacterium bovis in South Africa: are we losing the battle? Prev Vet Med. 2014;115:10-7.

45. Harmsen D, Dostal S, Roth A, Niemann S, Rothgänger J, Sammeth M, Albert J, Frosch M, Richter E. RIDOM: comprehensive and public sequence database for identification of Mycobacterium species. BMC Infect Dis. 2003;3:26.

46. Kumar S, Stecher G, Tamura K. MEGA7: Molecular evolutionary genetics analysis version 7.0 for bigger datasets. Mol Biol Evol. 2015:33:1870-74.

47. Saitou N, Nei M. The neighbor-joining method: a new method for reconstructing phylogenetic trees. Mol Biol Evol. 1987;4:406-25.

\section{Ready to submit your research? Choose BMC and benefit from:}

- fast, convenient online submission

- thorough peer review by experienced researchers in your field

- rapid publication on acceptance

- support for research data, including large and complex data types

- gold Open Access which fosters wider collaboration and increased citations

- maximum visibility for your research: over $100 \mathrm{M}$ website views per year

At BMC, research is always in progress.

Learn more biomedcentral.com/submissions 